\title{
Correction to: Theoretical prediction of radiation-enhanced diffusion behavior in nickel under self-ion irradiation
}

\author{
Xiao-Ya Chen ${ }^{1,2}$ (10) A-Li Wen ${ }^{2}$ Cui-Lan Ren ${ }^{2,3}$ (10 $\cdot$ Cheng-Bin Wang ${ }^{2,3}$ (1) \\ Wei Zhang ${ }^{2,3} \cdot$ He-Fei Huang ${ }^{2}$ (I) $\cdot$ Zhi-Wen Chen $^{1} \cdot$ Ping Huai $^{2,4,5}$
}

Published online: 17 September 2020

(C) China Science Publishing \& Media Ltd. (Science Press), Shanghai Institute of Applied Physics, the Chinese Academy of Sciences, Chinese Nuclear Society and Springer Nature Singapore Pte Ltd. 2020

\section{Correction to: NUCL SCI TECH (2020) 31:79 https://doi.org/10.1007/s41365-020-00791-w}

In the original publication, there is a mistake in Eq. (9) and its explanations (Sect. 2.2). The correction is as follows:

$D_{\mathrm{v}}=\alpha a_{0}^{2} v_{0} \exp \left(-H_{\mathrm{v}}^{m} / k_{\mathrm{B}} T\right)$

where $a_{0}$ is the lattice constant and $v_{0}$ is the attempt frequency for vacancy exchange $[1,27], k_{\mathrm{B}}$ is the Boltzmann's constant. For a fcc lattice, each atom has 12 nearest neighbors $(z=12)$, and the jump distance is $A a_{0}=\frac{1}{\sqrt{2}} a_{0}$. Then, we have $\alpha=1 / 6 z A^{2}=1$ [1].

The original article can be found online at https:// doi.org/10.1007/s41365-020-00791-w.

Cui-Lan Ren

rencuilan@sinap.ac.cn

$\triangle$ Zhi-Wen Chen

zwchen@shu.edu.cn

1 School of Environmental and Chemical Engineering, Shanghai University, Shanghai 200444, China

2 Shanghai Institute of Applied Physics, Chinese Academy of Sciences, Shanghai 201800, China

3 Key Laboratory of Interfacial Physics and Technology, Chinese Academy of Sciences, Shanghai 201800, China

4 Shanghai Synchrotron Radiation Facility, Shanghai Advanced Research Institute, Chinese Academy of Sciences, Shanghai 201204, China

5 School of Physical Science and Technology, ShanghaiTech University, Shanghai 201210, China
Furthermore, the production rate of freely migrating defects, $K_{0} / K$, used in this work (Table 1 ) should be 0.03 (assumed).

The authors would like to apologize for the inconvenience caused. 